\title{
DEMOCRACIA, CALIDAD DE LA DEMOCRACIA Y DEMOCRATIZACIÓN
}

\author{
DEMOCRACY, QUALITY OF DEMOCRACY AND \\ DEMOCRATIZATION
}

Osvaldo Iazzetta

\begin{abstract}
UFRGS CI ÊNCIA POLITTICA

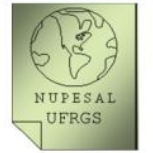

Reprodução e autorização: revistadebates@ufrgs.br ISSN Eletrônico 1982-5269 ISSN Impresso 2236-479X www.revistadebates.ufrgs.br
\end{abstract}

\begin{abstract}
Resumen
Los estudios sobre la democracia desarrollados en las últimas tres décadas han acompañado los climas y contextos cambiantes que sacudieron a la región. Si los problemas de la transición y consolidación de la democracia dominaron la agenda de investigación de los años ochenta, un lugar equivalente ocupan hoy los estudios en torno a la calidad de la democracia, tal como lo prueba la vasta literatura sobre el tema y la proliferación de experiencias que, en diferentes puntos de nuestra región, ensayan evaluaciones inspiradas en ella. El artículo sugiere que este creciente interés por la calidad de la democracia es también compartido por los estudios que abordan la problemática de la democratización, temas que aparecen, en no pocos textos relativos a esta cuestión, como inseparables y mutuamente conectados. En tanto la primera responde al interés por conocer y evaluar qué democracia tenemos, la segunda concibe a la construcción democrática como una empresa que no tiene término e incorpora nuevas tareas y aspiraciones que expresan la sensibilidad cambiante de nuestras sociedades y sus valores.
\end{abstract}

Palabras Clave: Democracia; Calidad de la Democracia; Democratización.

\begin{abstract}
Researches about democracy carried out during the last three decades have gone hand in hand with the changing contexts and conditions that shook the region. The problems with the transition and consolidation of the democracy dominated the agenda during the 80's and something similar is happening nowadays with researches on the quality of democracy. There are hundreds of studies carried out about this topic as well as experiences that try to evaluate the results. This article suggests that the raising attention on the quality of democracy is also shown in studies that deal with the issue of democratization, topics which are discussed in many texts as tethered and mutually connected with one another. While the former addresses the issue of knowing and assessing which democracy we have, the latter considers the democratic view as a unit that does not have an end and incorporates new tasks and challenges that express the changing sensibility of our societies and values.
\end{abstract}

Keywords: Democracy; Quality of Democracy; Democratization. 


\section{Del entusiasmo democrático al pesimismo de los 1990}

La recuperación de la democracia despertó en nuestra región un fuerte entusiasmo que se vio ampliamente reflejado en la literatura que acompañó su retorno. Sin embargo, al comenzar la década del 1990, esa sensación fue desplazada por un desencanto que surgía de constatar la persistencia de viejas prácticas y estilos políticos poco consonantes con la democracia imaginada.

Ese cambio de percepción no demoró en reflejarse en una nueva generación de estudios $^{1}$ que aportó nuevos diagnósticos, despojados de las ilusiones y voluntarismo que contenían algunos de los análisis sobre transición democrática ${ }^{2}$. La tensa convivencia entre rasgos democráticos y autoritarios que destacaban esos abordajes, derribó muchas de las expectativas fundacionales que acompañaron el retorno de la democracia y nos recordaron que en ellas aún latían legados y reflejos provenientes del pasado ${ }^{3}$.

Estos estudios nos permitieron disponer de una mejor comprensión de la complejidad que encierra todo proceso de construcción democrática, aceptando con mayor modestia, que esa tarea se encaraba con elementos novedosos (como la revalorización de las libertades civiles y derechos humanos) pero también con materiales viejos que confirmaban la permanencia de huellas culturales y prácticas de gobierno, poco afines con una democracia.

Ese desencanto pronto se tradujo en un ajuste conceptual que derivó en categorías híbridas o mixtas que intentaban captar la convivencia entre rasgos autoritarios y democráticos observada en muchos de los regímenes posautoritarios extendidos por la región.

En tanto conceptos como "democracias híbridas" (MALLOY, 1993) o "delegativas" (O’DONNELL, 1991) enfatizaban la coexistencia de procedimientos electorales democráticos y estilos decisorios autoritarios; otros, como "enclaves autoritarios" (GARRETÓN, 2000, p. $85)$, destacaban legados más severos - como los enfrentados por la democracia chilena postPinochet - que designaban la institucionalización de ciertos aspectos del régimen anterior e imponían límites a la soberanía popular y al Estado de Derecho.

Ya sea apelando a tipos híbridos o a subtipos disminuidos de democracia, estos nuevos estudios, ponían en evidencia la distancia que asomaba entre la democracia imaginada y la efectivamente lograda.

Al rescatar variables históricas y una perspectiva de largo plazo, estos enfoques aportaron una aproximación más realista sobre los procesos de democratización que, de ser

\footnotetext{
${ }^{1}$ Un interesante tratamiento sobre este giro puede hallarse en Peruzzotti (1998) y Torre (1995).

${ }^{2}$ Una valiosa reflexión sobre este cambio de actitud puede hallarse en las críticas a la idea de "consolidación democrática" formuladas por O’Donnell (1996; 1997; 2002).

${ }^{3}$ Los estudios más representativos de ese momento fueron realizados por O’Donnell (1991), Nino (1992), Malloy (1993), Weffort (1993), Torre (1991), Conaghan y Malloy (1997), Ducatenzeiler y Oxhorn (1994).
} 
concebidos como una construcción ex novo, pasaron a entenderse como una ardua y prolongada tarea que no era posible encarar ignorando las tradiciones y prácticas políticas preexistentes (TORRE, 1995).

Esa revisión también comprendió a la idea de consolidación, concebida inicialmente como un proceso secuencial que contemplaba un punto de llegada. La concepción teleológica que ella encierra se manifestó inadecuada para entender una empresa que no tiene término y permanece siempre expuesta al riesgo de invertir la ruta. La experiencia histórica indica que no existe democracia - incluso aquellas de mayor antigüedad- que pueda reposar en la confianza de que ha sido conquistada de una vez para siempre y que ese logro resultará irreversible.

A su vez, la ilusión de un punto terminal promueve un esclerosamiento de las energías y del compromiso que es preciso renovar cotidianamente para perfeccionarla, ignorando que ese proceso - abierto e inacabado - entraña una incesante búsqueda de nuevos horizontes y metas.

Una de las derivaciones más significativas de ese ajuste y cambio de estado anímico hoy podemos reconocerlo en la creciente atención que despiertan los estudios sobre calidad de la democracia y democratización, temas que aparecen, en no pocos textos relativos a esta cuestión, como inseparables y mutuamente conectados (VARGAS CULLELL, 2011; O’DONNELL, 2010).

La primera de estas cuestiones responde al interés por conocer y evaluar - siempre que las herramientas disponibles lo permitan - qué democracia tenemos; la segunda enfatiza que la construcción democrática es una empresa que no tiene término e incorpora nuevas tareas y aspiraciones que expresan la sensibilidad cambiante de nuestras sociedades y sus valores.

\section{La preocupación por la calidad de la democracia}

Los estudios sobre la democracia en las últimas tres décadas han acompañado los climas y contextos cambiantes que sacudieron a la región. Si los problemas de la transición y consolidación de la democracia - luego revisada y desechada por los autores que la impulsaron -, dominaron la agenda de investigación de los años ochenta, un lugar equivalente ocupan hoy los estudios en torno a la calidad de la democracia, tal como lo prueba la vasta literatura sobre el tema y la proliferación de experiencias que, en diferentes países de nuestra región, ensayan evaluaciones inspiradas en ella.

Que hoy resulte posible abordar esta cuestión, se debe entre otras razones, al hecho de que la mayoría de las democracias de la región disponen de un umbral básico - una poliarquía 
- que, aunque insuficiente, permite interrogarnos sobre lo que aún resta perfeccionar, por encima de ese piso conquistado.

La inquietud por la calidad de una democracia, puede hallarse embrionariamente contenida en autores que contribuyeron de manera decisiva a formular una definición minimalista de democracia. El interrogante lanzado por Dahl, ¿cuán democráticas son nuestras democracias? ha actuado como estímulo para algunas versiones y experiencias representativas de este enfoque (VARGAS CULLELL, 2003).

Esa simple y modesta pregunta acerca de la democraticidad de la democracia abrió un amplio y rico campo de indagación que aún debe lidiar, sin embargo, con ciertos desafíos conceptuales y operativos que incluyen no sólo cómo debe evaluarse una democracia sino principalmente, qué aspectos o dimensiones de la vida democrática son susceptibles de tal evaluación. Sobre esto aún no hay acuerdo - e ignoramos si alguna vez lo habrá - pues lo que está en discusión finalmente, es la propia materia a evaluar y calificar, esto es, la democracia.

Como bien nos recuerda Vargas Cullell (2011, p. 68) "la calidad de la democracia es un concepto derivado [...]" que está fuertemente atado al objeto que califica. Ello exige disponer previamente de un concepto de democracia claramente especificado pero asumiendo que no cualquier concepto se ajusta a la ambición que late en la idea de calidad de democracia. Ésta - aclara el autor -, contribuyó a ampliar la noción de democracia desbordando la comprensión limitada que caracterizó a los estudios centrados sólo en el régimen político (VARGAS CULLELL, 2011).

En suma, los estudios sobre calidad de la democracia no sólo exigen un concepto claramente definido sino también su ampliación, desafiando de ese modo los límites que la teoría democrática predominante ha venido aceptando como válidos.

El punto central de la controversia radica por consiguiente en la materia a definir, esto es, la democracia.

Pese a todos los avances y acuerdos mínimos que, desde Schumpeter a Dahl, se han acumulado en la búsqueda de una definición empírica, democracia es un concepto "esencialmente debatible" de modo tal que ninguna definición será “[...] consensuada para siempre o por completo" (O’DONNELL, 2010, p. 44).

Se trata - como destaca Whitehead (2011a) - de un concepto intemporal que si bien contiene principios necesarios e invariables, también expresa ideales y valores subjetivos que varían con el tiempo y el espacio, y dispone de una frontera exterior contextualmente cambiante.

En suma, hay dos elementos que distinguen a este concepto: su carácter esencialmente "debatible" (o disputable) y su inevitable variabilidad. 
La noción de democracia se inscribe dentro de lo que el filósofo británico W. B. Gallie definió como un "concepto esencialmente controvertible"4. Esta idea sobre la democracia ha sido luego recogida por Collier y Levitsky (1998) y también Whitehead (2011a; 2011b) la extiende al concepto "democratización", sugiriéndose en ambos casos que se trata de conceptos que encierran "[...] disputas interminables sobre sus empleos correctos por parte de los usuarios" (GALLIE, 1956, p. 169).

En cuanto a su inevitable variabilidad, O'Donnell (2010) recuerda que la democracia

[...] es el único tipo de arreglo político que inherentemente implica un horizonte normativa e históricamente abierto [...]. Esa proyección hacia un futuro interminable, indefinido, riesgoso y esperanzador es la razón de horizonte histórico abierto por la que debemos preferir la democracia. (O’DONNELL, 2010, p. 294-295, grifos nuestros).

En la idea de democracia existe por consiguiente, una tensión entre realidad y valores cambiantes. Como destaca Sartori (2003, p. 22), la democracia es un concepto "deontológico" de modo tal que lo "que la democracia es [...] no puede ser desligada de aquello que la democracia debería ser". La democracia es por tanto un concepto descriptivo como valorativo y su significado varía según las condiciones históricas y culturales y de la costumbre, que son maleables.

Ese carácter contingente se traduce en la constante tentación y necesidad de introducir nuevos elementos en la definición de democracia. Sin embargo, aunque existe un extendido consenso respecto a la necesidad de ampliar el concepto de democracia, aún representa un motivo de controversia definir qué aspecto o dimensión de la política o la sociedad debe incluirse dentro del mismo y por qué éstos son relevantes para la democracia. Incluso quienes adhieren a la necesidad de tal ampliación advierten, como Munck (2011, p. 25), que "[...] la adición de criterios que van más allá de la democracia electoral sea propuesta en términos conceptuales claros y sea justificada en sólidos fundamentos teóricos".

O’Donnell (2010) ha alentado una ampliación teóricamente disciplinada del concepto de democracia que no renuncie a investigar y conceptualizar sus complejas relaciones con el estado y la sociedad pero tampoco comprometa su operatividad y comparatividad. En sus textos de las últimas dos décadas, ha insistido en que los déficit de las democracias latinoamericanas no logran comprenderse a partir de un análisis centrado en el régimen

\footnotetext{
${ }^{4}$ Esta es la traducción de Gallie sugerida por Mazzuca (2006).

${ }^{5}$ Inspirándose en el mismo texto de Gallie, Lukes (2007, p. 65) también aplica esa idea al concepto de "poder", advirtiendo que "en verdad, meterse en tales disputas es meterse en política".
} 
político sino también exigen conocer el tipo de estado y de estructura social con las que aquellas conviven.

\section{La democratización y su reverso: la desdemocratización}

En su último trabajo O’Donnell (2010, p. 292-293) sostiene que la “[...] histórica variabilidad e indecidibilidad de diversos libertades y derechos [...]" prohíbe una rígida definición de la democracia, por lo que mantiene un carácter siempre abierto. Por esa razón, concluye

[...] que el objeto adecuado de reflexión, así como de la práctica política, reside más en la democratización que en la democracia. [...] Los grados y solidez de esas expansiones son medida, si bien difícil de calibrar empíricamente, del grado de democratización o calidad de las democracias en cada caso". (O’DONNELL, 2010, p. 292-293, grifos neustros).

Este desplazamiento del objeto y la práctica - desde la democracia a la democratización - nos incita a pensar en un trayecto que no tiene término y apuesta a que este impulso democratizador trascienda el momento electoral y se extienda a la sociedad y al Estado, en suma, que penetre nuestra experiencia social cotidiana ${ }^{6}$.

Asimismo, O’Donnell (2010, p. 196) advierte que estos procesos de democratización no sólo no tienen fin sino que son "[...] potencialmente reversibles: siempre es posible que sean interrumpidos [...]" e inviertan la ruta.

En un mismo sentido Tilly (2010, p. 29) nos recuerda que "la democratización es un proceso dinámico que siempre permanece incompleto y corre permanentemente el riesgo de inversión, de desdemocratización".

La experiencia histórica enseña que no existe democracia - incluso aquellas más antiguas - que pueda reposar en la confianza de que ha sido conquistada de una vez para siempre y que ese logro resultará irreversible. La "longevidad" de una democracia no parece representar una condición suficiente para asegurar su permanencia y perfeccionamiento.

\footnotetext{
${ }^{6}$ Cabe aclarar que la problemática de la democratización ya formaba parte de sus preocupaciones, como lo prueba el texto publicado en 1993 bajo el título: “Acerca del estado, la democratización y algunos problemas conceptuales. Una perspectiva latinoamericana con referencias a países poscomunistas" (O’DONNELL, 1993).

${ }^{7}$ Me apoyo en la presentación de Leonardo Avritzer realizada en el X Congreso Nacional de Democracia realizado en la Universidad Nacional de Rosario (3 al 7 de septiembre de 2012). Avritzer alega, por razones que comparto, que la "longevidad" no constituye un indicador confiable de calidad democrática (AVRITZER, 2012).
} 
Como señalamos para el caso de calidad de la democracia, la idea de democratización también es un concepto derivado y por ende está estrechamente atada a la noción de democracia que le da sustento. Por consiguiente, son variadas las formulaciones disponibles como así también las opciones para operacionalizar ese concepto.

Vargas Cullell (2011, p. 70) propone una definición - inspirada en Whitehead- que entiende a "[...] la democratización como ese proceso abierto por medio del cual la organización democrática de relaciones de poder se disemina en una sociedad, afectando los distintos ámbitos de la vida social”.

Una temprana formulación de esa misma expectativa ya podemos hallarla en $E l$ futuro de la democracia, libro en el que Bobbio (1994) señala que en adelante, los avances de la democracia deberán medirse por la conquista de espacios que aún no han sido afectados por el proceso de democratización ${ }^{8}$.

Si hoy se quiere tomar un indicador del desarrollo democrático, agrega Bobbio,

[...] éste ya no puede ser el número de personas que tienen derecho de votar, sino el número de las sedes, diferentes de las sedes políticas, en las cuales se ejerce el derecho de voto. Para dar un juicio sintético pero eficaz del grado de democratización de un determinado país, el criterio ya no debe ser el de 'quién' vota, sino el de 'dónde' vota (y que quede claro que aquí entiendo 'votar' como el acto típico y más común del participar, pero de ninguna manera pretendo limitar la participación al voto). (BOBBIO, 1994, p. 71).

En la versión de Tilly, el foco de la democratización está puesto en otro aspecto. Para este autor la democracia se presenta "[...] como una clase de relaciones entre Estados y ciudadanos, y democratización y desdemocratización consistirán en cambios en tales modalidades" (TILLY, 2010, p. 43).

De acuerdo a Tilly (2010), el grado de democracia depende de la medida en que el Estado se comporta de conformidad con las demandas expresadas por sus ciudadanos y la democratización - tanto como su reverso, la desdemocratización - puede evaluarse según aumente o disminuya dicha conformidad. Parte de la tarea del Estado consiste en "consultar" a los ciudadanos acerca de sus opiniones, necesidades y demandas pero ello exige evaluar al mismo tiempo, en qué medida, diferentes grupos de ciudadanos llegan a ver traducidas sus demandas en las prácticas y políticas públicas del Estado (TILLY, 2010). Este punto de

\footnotetext{
${ }^{8}$ Bobbio acepta que aunque deseable, esa meta parece lejana e incierta, especialmente cuando pensamos en centros de poder jerárquico y centralizados propios de una sociedad compleja (tales como la gran empresa y la administración pública). Entendida en un sentido amplio, como la ocupación de nuevos espacios dominados hasta ahora por organizaciones de tipo jerárquico o burocrático, este proceso expansivo deberá continuar con la democratización del Estado y la democratización de la sociedad (BOBBIO, 1994).
} 
partida adoptado por Tilly (2010) conduce a prestar preferente atención a las capacidades reunidas por el Estado para satisfacer esas demandas, convirtiendo a aquella en una dimensión decisiva del proceso de democratización ${ }^{9}$.

Otra versión - que comparte algunos aspectos con Tilly - podemos hallarla en uno de los textos seminales sobre la transición a la democracia (O'DONNELL y SCHMITTER, 2010) que al desarrollar el concepto de "democratización” presenta a la "ciudadanía" como el principio rector de la democracia.

Tal como ambos autores la definieron entonces, la democratización es concebida como un triple proceso expansivo que se manifiesta:

a. extendiendo los procedimientos democráticos a áreas e instituciones regidas hasta entonces por otros principios;

b. expandiendo derechos y obligaciones a individuos que antes no gozaban de los mismos;

c. abarcando ámbitos e instituciones públicas y privadas que no estaban abiertas a la participación ciudadana.

Esta idea de democratización, entendida como la propagación o difusión de principios democráticos a diferentes ámbitos de la vida y práctica social, avanza más allá de la noción de "democracia electoral" que restringe a la democracia a los procedimientos activados en el momento electoral. El conjunto de derechos y garantías que hacen posible elecciones periódicas, competitivas y libres de coacción, son una condición necesaria para hablar de democratización pero de ningún modo ésta se agota en ese instante fugaz, por crucial que resulte para una democracia.

Estos aportes contienen enormes potencialidades y coinciden en presentar a la democratización como un proceso expansivo - no terminal - (ACKERMAN, 2006) que desborda esa noción limitada de democracia e invita a ampliar sus alcances, más allá de la "democracia electoral" o de la noción de "poliarquía".

La expectativa de una propagación de los principios democráticos que hoy hallamos reflejada en una amplia literatura (ACKERMAN, 2006; VARGAS CULLELL, 2008), ha estado presente en ciertos textos decisivos de los 1980, como señalamos hace un momento al describir el concepto formulado por O’Donnell y Schmitter (2010) o al recordar textos como el de Bobbio (1994).

\footnotetext{
${ }^{9}$ Como contrapartida, Tilly (2010, p. 44) sostiene que "[...] el predominio de las formas no estatales de poder afecta fuertemente a las posibilidades de democratización".
} 
Ahora bien, entre los estudios sobre democratización de los años 1980 y los más recientes, lo que contrasta es que los trabajos de aquella primera etapa aún estaban impregnados de un cierto optimismo que llevaba a concebirla como un proceso que, una vez iniciado, conduciría, con algunos pocos inconvenientes y problemas, a la democracia. Ellos aún no eran capaces de percibir todo el drama de la lucha por la democracia que incluye experiencias de democratización fallida y regresiones autoritarias, tal como mostraron muchos procesos posteriores (PASQUINO, 2011).

Los trabajos recientes ya no adolecen de esa tentación optimista y unilineal - en parte por las evidencias de fracasos y retrocesos acumulados en este lapso - y no son pocas las versiones que introducen una idea más compleja de democratización, admitiendo, como su contracara inseparable, el riesgo potencial de regresión y reversión de la ruta transitada.

La idea de que estos procesos son "potencialmente reversibles" - véase O’Donnell (2010) -, o la noción de desdemocratización - en Tilly (2010) y Bovero (2010) -, son apenas expresión de este cambio de paradigma que tiende a abandonar el simplismo y optimismo que invita a superar Pasquino (2011).

Desde luego es preciso avanzar hacia una formulación del concepto de modo tal que permita una mejor traducción operativa para encarar futuras investigaciones. De todos modos, entendida en un sentido amplio, la idea de democratización comprende tanto la extensión de derechos a quienes estaban privados de ellos, la inclusión de individuos o actores que permanecían excluidos de la participación como así también, la difusión de los principios democráticos a ámbitos no regidos hasta entonces por esos criterios. Concebida de este modo, ella se presenta como un concepto multidimensional que contiene enormes potencialidades para evaluar las variaciones - en un sentido favorable o desfavorable - de las complejas relaciones entre democracia, estado y ciudadanía.

Osvaldo Iazzetta é Doutor em Ciências Sociais pela Flacso (Brasil) e pela Universidade de Brasília, professor e investigador da Facultad de Ciencia Política y Relaciones Internacionales da Universidad Nacional de Rosario (Argentina).

E-mail: iazzetta@arnet.com.ar

\section{Referencias}

ACKERMAN, John M. Democratización: pasado, presente y futuro. Perfiles Latinoamericanos, México, s./v., n. 28, p. 117-157, jul.-dic. 2006. 
AVRITZER, Leonardo. Sociedad civil y calidad de la democracia en América Latina. In: Congresso Nacional de Democracia. Rosário, Argentina, 5., 2012.

BOBBIO, Norberto. El futuro de la democracia. Barcelona: Planeta-Agostini, 1994.

BOVERO, Michelangelo. La democracia y sus condiciones. Revista de la Facultad de Derecho de México, Cidade do México, tomo LX, n. 253, p. 11-29, jan.-jun. 2010.

COLLIER, David; LEVITSKY, Steven. Democracia con adjetivos. Innovación conceptual en la investigación comparativa. Ágora - Cuadernos de Estudios Políticos, Buenos Aires, año 4, n. 8, p. 99-122, verano, 1998.

CONAGHAN, Catherine; MALLOY, James. Democracia y neoliberalismo en Perú, Ecuador y Bolivia. Desarrollo Económico, Buenos Aires, v. 36, n. 144, p. 867-890, ene.-mar. 1997.

DUCATENZEILER, Graciela; OXHORN, Philip. Democracia, autoritarismo y el problema de la gobernabilidad en América Latina. Desarrollo Económico, Buenos Aires, v. 34, n. 133, p. 31-52, abr.-jun. 1994.

GALLIE, Walter Bryce. Essentially Contested Concepts. In: Meetingo of the Aristotelian Society at 21. London, 1956.

GARRETÓN, Manuel Antonio. Política y sociedad entre dos épocas. América Latina en el cambio de siglo. Rosário: Homo Sapiens, 2000.

LUKES, Steven. El poder. Un enfoque radical. Madrid: Siglo XXI, 2007.

MAZZUCA, Sebastián. Ampliando las concepciones sobre la democracia: acceso al poder versus ejercicio de poder. In: ALARCÓN OLGUÍN, Victor (Coord.). Metodologías para el análisis político. Enfoques, procesos e instituciones. México D.F.: Universidad Autónoma Metropolitana (UAM), Sede Iztapalapa, 2006. p. 279-308.

MUNCK, Gerardo. Los estándares de la democracia: hacia una formulación de la cuestión democrática en América Latina. Journal of Democracy en Español, Santiago, v. 3, p. 22-41, jul. 2011. 
MALLOY, John. Política económica e o problema da gobernabilidade democrática nos Andes Centrais. In: SOLA, Lourdes Sola (Comp.). Estado, mercado e democracia. Política e economia comparadas. Río de Janeiro: Paz e Terra, 1993. p. 99-126.

NINO, Carlos S. Un país al margen de la ley. Buenos Aires: Emecé, 1992.

O’DONNELL, Guillermo. Democracia delegativa. Novos Estudos CEBRAP, São Paulo, v. 31, n. 92, p. 25-40, out. 1991.

. Acerca del estado, la democratización y algunos problemas conceptuales. Una perspectiva latinoamericana con referencias a países poscomunistas. Desarrollo Económico, Buenos Aires, v. 33, n. 130, p. 163-184, jul.-set. 1993.

. Otra institucionalización. Ágora - Cuaderno de Estudio Político, Buenos Aires, s./v., n. 5, p. 5-28, invierno, 1996.

. Ilusiones y errores conceptuales. Ágora - Cuaderno de Estudio Político, Buenos Aires, s./v., n. 6, p. 209-221, verano, 1997.

. Ilusiones sobre la consolidación. Nueva Sociedad, Caracas, s./v., n. 144, p. 70-89, jul.-oct. 2002.

. Democracia, agencia y estado. Teoría con intención comparativa. Buenos Aires: Prometeo, 2010.

O’DONNELL, Guillermo; Philippe SCHMITTER. Transiciones desde un gobierno autoritario/4: Conclusiones tentativas sobre democracias inciertas. Buenos Aires: Prometeo, 2010.

PASQUINO, Gianfranco. Orden político y democratización. Revista SAAP, Buenos Aires, v. 5, n. 2, p. 423-435, nov. 2011.

PERUZZOTTI, Enrique. La naturaleza de la nueva democracia argentina. Revisando el modelo de democracia delegativa. In: URZÚA, Raúl; AGÜERO, Felipe (eds.). Fracturas en la gobernabilidad democrática. Santiago de Chile: Centro de Análisis de Políticas Públicas, 1998. p. 289-314. 
SARTORI, Giovanni. ¿Qué es la democracia? Buenos Aires: Taurus, 2003.

TILLY, Charles. Democracia. Madrid: Akal, 2010.

TORRE, Juan C. América Latina. El gobierno de la democracia en tiempos difíciles. Revista de Estudios Políticos, Madrid, s./v., n. 74, p. 145-161, oct.-dic. 1991.

De la utopía democrática a la democracia argentina. Agora - Cuaderno de Estudios Políticos, Buenos Aires, v. 01, n. 2, p. 117-181, verano, 1995.

VARGAS CULLELL, Jorge. Auditorías ciudadanas sobre la calidad de la democracia: una herramienta para la identificación de desafíos democráticos. In: O’DONNELL, Guillermo; IAZZETTA, Osvaldo; VARGAS CULLELL, Jorge (comps.). Desarrollo Humano y Ciudadanía: Reflexiones sobre la calidad de la democracia en América Latina. Rosário: Homo Sapiens/PNUD, 2003. p. 149-221.

. Democratización y calidad de la democracia. In: OCHOA, Oscar (Comp.). La reforma del estado y la calidad de la democracia en México: una reflexión sobre sus instituciones. México: Escuela de Graduados en Administración Pública del Instituto Tecnológico de Monterrey, 2008.

. La calidad de la democracia y el estudio comparado de la democratización. Revista

Latinoamericana de Política Comparada, Quito, v. 5, p. 67-94, jul. 2011.

WEFFORT, Francisco C. ¿Cuál democracia? San José: FLACSO, 1993.

WHITEHEAD, Laurence. Democratización: Teoría y experiencia. México: Fondo de Cultura Económica, 2011a.

. Un concepto de democratización reanimado: la metáfora biológica. Revista SAAP, Buenos Aires, v. 5, n. 2, p. 401-419, nov. 2011b.

Texto recebido em $02 / 10 / 2012$.

Aprovado em 10/01/2013. 